\title{
Pertuzumab As Second- or Later-Line Therapy For Human Epidermal Growth Factor Receptor 2- Positive Metastatic Breast Cancer: A Swiss Clinical Experience
}

\section{Ewelina Biskup ( $\square$ ewelinabiskup@yahoo.de)}

Shanghai University of Medicine and Health Sciences https://orcid.org/0000-0002-9871-927X

Céline Montavon Sartorius

University Hospital Basel

Andreas Müller

Kantonsspital Winterthur

Cornelia Leo

Kantonsspital Baden

Catrina Uhlmann Nussbaum

Kantonsspital Olten

Elena Laura Georgescu Margarint

Shanghai East International Medical Center

Daniel Koychev

Tumorzentrum ZeTuP: Tumor und Brustzentrum ZeTuP

Alexander Schreiber

Kantonsspital Aarau

Christian Taverna

Kantonsspital Muensterlingen

David Thorn

Oncology Private Practice Basel, Affiliate of the Department of medical Oncology, University Hospital Basel, Basel

\section{Marcus Vetter}

Medical University Clinic, Canton Hospital Baselland, Liestal; University of Basel, Liestal

\section{Research Article}

Keywords: metastatic breast cancer, pertuzumab, trastuzumab, human epidermal growth factor receptor 2 , second-line therapy, overall survival

Posted Date: November 10th, 2021 
DOI: https://doi.org/10.21203/rs.3.rs-1051053/v1

License: (c) (1) This work is licensed under a Creative Commons Attribution 4.0 International License. Read Full License 


\section{Abstract}

Background: Trastuzumab and pertuzumab with taxane-based chemotherapy are considered the first-line standard therapy for human epidermal growth factor receptor 2 (HER2)-positive metastatic breast cancer $(\mathrm{mBC})$. Pertuzumab is also a later-line therapy for $\mathrm{mBC}$ in Switzerland, although limited safety and efficacy data are available. This study assesses the therapeutic regimens, toxicities, and clinical outcomes after second- or later-line pertuzumab therapy in patients with $\mathrm{mBC}$ who did not receive pertuzumab as a first-line therapy.

Materials and Methods: Physicians from major Swiss oncology centers retrospectively completed a questionnaire for each pertuzumab-naive patient who was treated with pertuzumab as a second- or laterline therapy.

Results: Of 35 patients with HER2-positive mBC (median age: 49 years; range: 35-87 years), 14 received pertuzumab as a second-line therapy, six as a third-line therapy, and 15 as a fourth- or later-line therapy. Twenty patients (57\%) died during the study period. The median overall survival was 74.2 months ( $95 \%$ confidence interval: 47.6-139.8 months). Grade (G) 3/4 adverse events (AEs) were reported in $14 \%$ of patients, with only one patient discontinuing therapy due to pertuzumab-related toxicities. The most common AE was fatigue (overall: 46\%; G3: $11 \%$ ). Overall, congestive heart disease occurred in $14 \%$ of patients (G3: 6\%), nausea in 14\% (all G1), and myelosuppression in 12\% (G3: 6\%).

Conclusion: The median overall survival of second- or later-line pertuzumab is similar to that reported for first-line pertuzumab, and the safety profile was acceptable. These data support using pertuzumab for second- or later-line therapy when it was not administered as first-line therapy.

\section{Introduction}

The human epidermal growth factor receptor 2 (HER2) proto-oncogene regulates cell growth, survival, and differentiation. HER2 overexpression, commonly referred to as HER2 positivity, is a poor prognostic sign in patients with breast cancer [1,2], and HER2-positive breast cancer accounts for $15 \%-20 \%$ of annual breast cancer-associated deaths. However, the introduction of anti-HER2 agents has revolutionized the standard of care for patients with HER2-positive breast cancer.

Pertuzumab is a recombinant human immunoglobulin $\mathrm{G}(\mathrm{IgG})$ monoclonal antibody that targets HER2 and acts by blocking HER2 dimerization with other HER family members including HER1, HER3, and HER4[1]. Trastuzumab is another targeted therapy that binds similarly to HER2. However, while pertuzumab binds to subdomain II of the HER2 extracellular domain epitope, trastuzumab binds to subdomain IV [1]. Although these agents bind to different HER2 epitopes, they have complementary mechanisms of action. The combined administration of trastuzumab and pertuzumab offers a more comprehensive blockade of the HER2 signaling pathway and results in more antitumor activity [1]. 
The CLEOPATRA phase III trial demonstrated the efficacy of adding pertuzumab to trastuzumabdocetaxel combination therapy as a first-line agent in patients with HER2-positive metastatic breast cancer $(\mathrm{mBC})$. The trial revealed that adding pertuzumab resulted in a significant improvement in overall survival (OS) compared with trastuzumab and docetaxel combined with a placebo [4,5]. In addition, the toxicity profiles of patients receiving pertuzumab and those receiving placebo were similar and manageable. Based on the significantly improved OS in the CLEOPATRA trial, the combination of pertuzumab, trastuzumab, and docetaxel has become the standard of care for first-line therapy for patients with $\mathrm{mBC}$. However, docetaxel is associated with significant toxicity. Therefore, several studies have reported that the combination of pertuzumab-trastuzumab with other chemotherapy agents, including weekly paclitaxel or nanoparticle albumin-bound (nab)-paclitaxel, is effective and more tolerable than regimens that include docetaxel [4-6]. Therefore, the combination of pertuzumabtrastuzumab with either taxane- or vinorelbine-based chemotherapy agents is the new standard first-line therapy for patients with HER2-positive mBC.

In patients with HER2-positive $\mathrm{mBC}$ who have recurrence or disease progression following first-line therapy with pertuzumab-trastuzumab, physicians may choose to re-target the HER2 receptor, though data regarding the safety and efficacy of this practice are relatively limited. Therefore, this retrospective, observational study involving physicians from major Swiss oncology centers aimed to assess the therapeutic regimens, toxicities, and clinical outcomes following second- or later-line pertuzumab therapy in patients with $\mathrm{mBC}$ who did not receive pertuzumab as a first-line chemotherapy agent.

\section{Materials \& Methods}

\section{Data Source}

Patients with HER2-positive $\mathrm{mBC}$ who received second- or later-line pertuzumab therapy without having received pertuzumab as a first-line therapy at nine major Swiss oncology centers were retrospectively identified. The patients' demographic, clinical, and therapeutic data were extracted from the medical records. Physicians of these patients were asked to complete a questionnaire regarding treatment regimens, safety, and survival for each included patient. The questionnaire was previously developed by one of the authors with the support of a statistical team.

\section{Patient Selection Criteria}

Pertuzumab-naive patients with HER2-positive $\mathrm{mBC}$ who had a relapse of $\mathrm{mBC}$ following first-line therapy between 2001 and 2016 and were subsequently treated with at least one dose of second- or later-line pertuzumab therapy were included in this study. Only female patients aged $\geq 18$ years who were treated at one of the included Swiss oncology centers were included. All eligible patients had mBC with known HER2-positive status. The study end-date was September 12, 2017. Patients who were male, diagnosed or treated for another primary cancer during the study period, or enrolled in other clinical trials and those who had previously been administered pertuzumab as a first-line treatment were excluded. 


\section{Clinical Study Measures}

The initial date of pertuzumab administration was defined as the index date. The primary endpoint was OS, calculated from the index date to death or the date of last follow-up. Data regarding disease progression, adverse events (AEs), and co-administered treatments were retrieved from the medical records. AEs were assessed according to the National Cancer Institute Common Terminology Criteria for Adverse Events ( $\mathrm{NCl} \mathrm{CTCAE,} \mathrm{version} \mathrm{4.0).} \mathrm{The} \mathrm{duration} \mathrm{of} \mathrm{pertuzumab} \mathrm{therapy} \mathrm{was} \mathrm{defined} \mathrm{as} \mathrm{the} \mathrm{time}$ from the index date to the date of the last administration of pertuzumab, death, or the end of the study. Data of patients who were alive or lost to follow-up at the end of the study were censored.

\section{Statistical Analyses}

Descriptive statistics were used to summarize patient demographics, clinical characteristics, treatment patterns, and AEs. Categorical variables are presented as frequency and percentage, and continuous variables are presented as mean and standard deviation or median and range.

A survival analysis was performed using the Kaplan-Meier methodology. All statistical analyses were performed using SPSS 17.0 statistical software (manufacturer name, location). Statistical significance was set at $p<0.05$.

\section{Ethics}

This retrospective, observational, questionnaire study was conducted according to the Declaration of Helsinki and local legislation. Due to the retrospective design, written informed patient consent was not required for this non-interventional study as the analysis used anonymous clinical data.

\section{Results}

\section{Patient Characteristics}

Overall, 35 female patients (median age: 49 years; range: 35-87 years) with HER2-positive mBC were included in the study. The clinicopathological characteristics of the patients are summarized in Table 1. Overall, 33 patients (94\%) had invasive ductal carcinoma and two $(6 \%)$ had invasive lobular carcinoma. Twenty-four patients (69\%) had ER-positive or PR-positive tumors, 17 (49\%) had stage IV cancer, and five $(14 \%)$ had stage III cancer at diagnosis. Sixteen patients (46\%) received trastuzumab therapy prior to pertuzumab therapy. The most common metastatic sites were the bone $(n=22 ; 63 \%)$, liver $(n=15 ; 43 \%)$, and lymph nodes $(n=14 ; 43 \%)$. 
Table 1

Patient characteristics

\begin{tabular}{|ll|}
\hline & Patients \\
\hline Histological subtype & \\
\hline Invasive ductal carcinoma & $33(94)$ \\
\hline Invasive lobular carcinoma & $2(6)$ \\
\hline Estrogen and/or progesterone receptor positive & $24(69)$ \\
\hline Stage IV cancer at first diagnosis & $17(49)$ \\
\hline Prior (neo)adjuvant chemotherapy & $15(43)$ \\
\hline Prior trastuzumab & $16(46)$ \\
\hline Metastatic sites & $22(63)$ \\
\hline Bone & $15(43)$ \\
\hline Liver & $15(43)$ \\
\hline Lymph nodes & \\
\hline Data are presented as $\mathrm{n}(\%)$. & \\
\hline
\end{tabular}

Ten patients (29\%) underwent primary breast-conserving procedures and $16(46 \%)$ underwent ablative (mastectomy) procedures. Thirteen patients (37\%) underwent radiotherapy and 15 (43\%) underwent chemotherapy.

\section{Treatments}

A total of 14 patients (40\%) received pertuzumab as a second-line agent, six (17\%) as a third-line agent, and $15(43 \%)$ as a fourth- or later-line agent (Table 2$)$. 
Table 2

Chemotherapy regimens

\begin{tabular}{|lllll|}
\hline & $\begin{array}{l}\text { All patients } \\
(\mathbf{n = 3 5 ) ( \% )}\end{array}$ & $\begin{array}{l}\text { Second-line } \\
(\mathbf{n = 1 4})\end{array}$ & $\begin{array}{l}\text { Third-line } \\
(\mathbf{n = 6})\end{array}$ & $\begin{array}{l}\text { Fourth-or later- } \\
\text { line }(\mathbf{n = 1 5})\end{array}$ \\
\hline $\begin{array}{l}\text { Pertuzumab + trastuzumab } \\
\text { chemotherapy }\end{array}$ & $29(83)$ & $12(86)$ & $5(83)$ & $12(80)$ \\
\hline Taxane & $19(54)$ & $8(57)$ & $4(67)$ & $7(47)$ \\
\hline Vinorelbine & $6(17)$ & $3(21)$ & $1(17)$ & $2(13)$ \\
\hline Gemcitabine & $1(3)$ & 0 & 0 & $1(7)$ \\
\hline Carboplatin & $1(3)$ & 0 & 0 & $1(7)$ \\
\hline Anthracycline & $2(6)$ & $1(7)$ & 0 & $1(7)$ \\
\hline $\begin{array}{l}\text { Pertuzumab + trastuzumab + } \\
\text { endocrine therapy }\end{array}$ & $1(3)$ & $1(7)$ & 0 & 0 \\
\hline $\begin{array}{l}\text { Pertuzumab + trastuzumab } \\
\text { alone }\end{array}$ & $5(14)$ & $1(7)$ & $1(17)$ & $3(20)$ \\
\hline Data are presented as $\mathrm{n}$ (\%). & & & & \\
\hline
\end{tabular}

The median duration of pertuzumab administration was 6 months (range: 2-60 months). The median duration of pertuzumab therapy was 5.5 months (range: 2-30 months) for patients who received pertuzumab as a second-line agent $(n=24), 6$ months (range: 2-60 months) for patients who received pertuzumab as a third-line agent $(n=6)$, and 11 months (range: 2-40 months) for patients who received pertuzumab as a fourth- or later-line agent $(n=15)$.

Most patients $(n=29 ; 83 \%)$ received a combination of pertuzumab-trastuzumab with another chemotherapeutic agent, including taxane $(n=19 ; 54 \%)$, vinorelbine $(n=6 ; 17 \%)$, anthracyclines $(n=2$; $6 \%$ ), and gemcitabine and carboplatin $(n=1 ; 3 \%)$. Among the remaining six patients, one (3\%) was administered pertuzumab-trastuzumab with endocrine therapy and five (14\%) were administered pertuzumab-trastuzumab alone.

Pertuzumab was discontinued mainly due to disease progression $(n=23 ; 66 \%)$ and toxicity $(n=5 ; 14 \%)$ (Figure 1). However, pertuzumab-associated toxicities were noted in one patient (3\%).

\section{Safety Outcomes}

G3 toxicities were reported in five patients (14\%) (Table 3). No patients had G4 toxicities. The most commonly-recorded AE was fatigue (overall: $n=16,46 \%$; G3: $n=4,11 \%$ ), followed by congestive heart failure (overall: $n=5,14 \%$; G3: $n=2,6 \%$ ), nausea (overall: $n=5,14 \% ; G 3: n=0$ ), and myelosuppression (overall: $n=4,11 \%$; $33: n=2,6 \%$ ). 
Table 3

Adverse events

\begin{tabular}{|llllll|}
\hline & All grades & Grade 1 & Grade 2 & Grade 3 & Grade 4 \\
\hline Fatigue & $16(46)$ & $7(20)$ & $5(14)$ & $4(11)$ & 0 \\
\hline Congestive heart disease & $5(14)$ & $1(3)$ & $2(6)$ & $2(6)$ & 0 \\
\hline Nausea & $5(14)$ & $5(14)$ & 0 & 0 & 0 \\
\hline Myelosuppression & $4(11)$ & $1(3)$ & $1(3)$ & $2(6)$ & 0 \\
\hline Diarrhea & $3(9)$ & $3(9)$ & 0 & 0 & 0 \\
\hline Vomiting & $1(3)$ & 0 & $1(3)$ & 0 & 0 \\
\hline Mucositis & $1(3)$ & 0 & $1(3)$ & 0 & 0 \\
\hline Data are presented as n (\%). & & & & \\
\hline
\end{tabular}

\section{Overall Survival}

At the final follow-up, 20 patients (57\%) had died. The median OS was 74.2 months ( $95 \%$ confidence interval: 47.6-139.8 months) (Figure 2).

\section{Discussion}

HER2-positive breast cancer has been linked to more aggressive tumor behavior and poorer outcomes than HER2-negative breast cancer [4]. However, the use of trastuzumab significantly improves the OS in patients with advanced HER2-positive breast cancer. Several new chemotherapeutic agents are currently being developed or are undergoing clinical investigation, including trastuzumab-emtansine, trastuzumabderuxtecan, neratinib, and tucatinib. Most patients with $\mathrm{mBC}$ experience disease progression following first-line treatment. The continuation of trastuzumab administration in patients who experience disease progression has been associated with improvement in the time to progression without an increased risk of treatment-related toxicity [4]. In 2010, a phase II trial conducted by Baselga et al. revealed that patients with $\mathrm{mBC}$ who experienced disease progression during prior trastuzumab therapy tolerated and responded well to the addition of pertuzumab to their therapeutic regimen [4] Combining pertuzumabtrastuzumab with taxane-based chemotherapy has become the new standard first-line therapy for patients with HER2-positive $\mathrm{mBC}$ based on the findings of the CLEOPATRA trial [7]. The combination of pertuzumab-trastuzumab with vinorelbine-based chemotherapy is as effective as and less toxic than the combination with taxane-based therapy [8]. However, as a second-line treatment, the administration of pertuzumab-trastuzumab combined with capecitabine was not superior to the combination of soletrastuzumab and capecitabine [4].

In the CLEOPATRA trial, the median OS was significantly higher in patients who received pertuzumab compared to patients who received placebo [7]. These previous results are consistent with the results of 
this study. In this study, the first dose of later-line pertuzumab was administered on the OS index date, rather than on the first treatment date for $\mathrm{mBC}$. Therefore, these results may reflect a patient selection bias.

As in the CLEOPATRA trial, chemotherapy accounted for most of the AEs reported in this study. Symptoms that developed during pertuzumab therapy in this study did not differ from those reported in previous studies. The addition of pertuzumab to the patients' therapeutic regimens did not increase cardiac toxicity in this study.

The pertuzumab-trastuzumab combination has a higher anti-cancer activity than either drug alone $[5,15]$. The combination therapy has been reported as effective against advanced breast cancer following disease progression [6] and for patients receiving neoadjuvant therapy. Pertuzumab, trastuzumab, and docetaxel have been associated with higher complete response rates in pathological samples than trastuzumab-docetaxel, pertuzumab-docetaxel, or pertuzumab-trastuzumab combinations [4]. The combination of pertuzumab-trastuzumab and chemotherapy has been approved for the neoadjuvant treatment of HER2-positive early breast cancer with a high risk of recurrence by the Food and Drug Administration in the United States and the Swiss Medic in Switzerland [4]. Therefore, an increasing number of patients with metastatic HER2-positive breast cancer will be pretreated with pertuzumab in the future.

More clinical uses of combination therapy are currently being investigated. A randomized phase III trial (Detect V/CHEVENDO; NCT02344472) comparing the safety and efficacy of the pertuzumab-trastuzumab combination with either endocrine therapy or chemotherapy is being conducted in patients with hormone receptor-positive and HER2-positive mBC. HER2-/neu-targeted combinations may help patients avoid potential chemotherapy-related toxicities while achieving high efficacy, which will improve the patients' quality of life.

This study has several limitations, including its retrospective nature and small sample size. In addition, the OS outcomes may have been overestimated as the index date for the OS calculation was much later in the disease course in this study compared to that in previous studies. This difference may have resulted in a patient selection bias toward favorable prognosis factors (long-term response in first-line therapy), similar to the bias in a previous study regarding trastuzumab-deruxtecan[17]. However, the remarkably long OS observed in this study warrants further prospective investigations of the use of pertuzumab in later-line regimens.

\section{Conclusion}

The median OS and safety profile of second- or later-line pertuzumab therapy are consistent with those reported for the first-line use of pertuzumab. These results support the use of pertuzumab in later-line therapy, though further validation in randomized controlled trials is necessary. 


\section{Declarations}

\section{(7) Acknowledgments}

We would like to thank Dr. Yongchao Wang and Dr. Jiayu Yao of State Key Laboratory of Oncogenes and Related Genes, Shanghai Cancer Institute, Department of Oncology, Renji Hospital, School of Medicine, Shanghai Jiao Tong University, Shanghai, China for their support during the submission process.

\section{Funding}

The study was funded by F. Hoffmann-La Roche Ltd., Basel, Switzerland.

Roche Pharma (Switzerland) provided administrative support for the analysis.

\section{Declarations of conflicts of interest}

Andreas Müller: Honoraria: Roche Switzerland, Novartis, Pfizer, Amgen, and Tesaro; Consulting/advisory role: Roche Switzerland, Novartis, AstraZeneca, Pfizer, and Amgen; Expert testimony: Roche Switzerland; Travel/accommodation expenses: Roche, AstraZeneca, Pfizer, and Novartis.

Cornelia Leo: Honoraria: Pfizer and AstraZeneca; Consulting/advisory role: Pfizer and AstraZeneca; Travel/accommodation expenses: Roche.

Catrina Uhlmann Nussbaum: Stock: Roche; Consulting/advisory role: Sanofi, Boehringer Ingelheim, and Roche.

Alexander Schreiber: Honoraria: Amgen, Roche, Pfizer, MSD, BMS, Lilly, Celgene, and Merck; Travel/accommodation expenses: Amgen, Roche, Pfizer, MSD, BMS, Lilly, Celgene, and Merck.

Christian Taverna: Consulting/advisory role: Celgene, Amgen, and Janssen; Research funding: Celgene.

David Thorn: Honoraria: Roche; Consulting/advisory role: Roche; Travel/accommodation expenses: Roche, Amgen, Teva, Novartis, and Celgene.

Markus Vetter: Employment: Roche (family member); Honoraria: Roche, Novartis, and Pfizer; Consulting/advisory role: Roche, Novartis, and Pfizer; Research funding: Roche.

Ewelina Biskup was supported by Krebsliga Schweiz, BIL KFS 4261-08-2017.

All other authors have no conflicts of interest to declare.

\section{References}

1. Slamon DJ, Clark GM, Wong SG et al. Human Breast Cancer: Correlation of Relapse and Survival With Amplification of the HER-2/neu Oncogene. Science. 1987;235:177- 
182 https://doi.org/10.1126/science.3798106

2. Andrulis IL, Bull SB, Blackstein ME et al. Neu/erbB-2 amplification identifies a poor-prognosis group of women with node-negative breast cancer. Toronto Breast Cancer Study Group. J Clin Oncol 1998;16:1340-1349. https://doi.org/10.1200/jco.1998.16.4.1340

3. Adams CW, Allison DE, Flagella K et al. Humanization of a recombinant monoclonal antibody to produce a therapeutic HER dimerization inhibitor, pertuzumab. Cancer Immunol Immunother. 2006;55:717-727. https://doi.org/10.1007/s00262-005-0058-x

4. Hudis CA. Trastuzumab--mechanism of action and use in clinical practice. $\mathrm{N}$ Engl $\mathrm{J}$ Med. (2007;357:39-51.) https://doi.org/10.1056/NEJMra043186

5. Scheuer W, Friess T, Burtscher H, Bossenmaier B, Endl J, Hasmann M. Strongly enhanced antitumor activity of trastuzumab and pertuzumab combination treatment on HER2-positive human xenograft tumor models. Cancer Res 2009;69:9330-9336. https://doi.org/10.1158/0008-5472.can-08-4597

6. Baselga J, Cortes J, Kim S-B, et al. Pertuzumab plus trastuzumab plus docetaxel for metastatic breast cancer. N Engl J Med. 2012;366:109-119. https://doi.org/10.1056/nejmoa1113216

7. Swain SM, Baselga J, Kim S-B et al. Pertuzumab, trastuzumab, and docetaxel in HER2-positive metastatic breast cancer. N Engl J Med. 2015;372:724-734.

https://doi.org/10.1056/nejmoa1413513

8. Bachelot T, Ciruelos E, Schneeweiss A et al. Preliminary safety and efficacy of first-line pertuzumab combined with trastuzumab and taxane therapy for HER2-positive locally recurrent or metastatic breast cancer (PERUSE). Ann Oncol. 2019;30:766-773. https://doi.org/10.1158/1538-7445.sabcs16p4-21-04

9. Perez EA, López-Vega JM, Petit T et al. Safety and efficacy of vinorelbine in combination with pertuzumab and trastuzumab for first-line treatment of patients with HER2-positive locally advanced or metastatic breast cancer: VELVET Cohort 1 final results. Breast Cancer Res. 2016;18:126. https://doi.org/10.1186/s13058-016-0773-6

10. Andersson M, López-Vega JM, Petit T et al. Efficacy and safety of pertuzumab and trastuzumab administered in a single infusion bag, followed by vinorelbine: VELVET Cohort 2 final results. Oncologist 2017;22:1160-1168. https://doi.org/10.1634/theoncologist.2017-0079

11. Biserni GB, Engstrom MJ, Bofin AM (2016) HER2 gene copy number and breast cancer-specific survival. Histopathology 69:871-879. https://doi.org/10.1111/his.13010

12. Waddell T, Kotsori A, Constantinidou A et al (2011) Trastuzumab beyond progression in her2-positive advanced breast cancer: the Royal Marsden experience. Br J Cancer 104:1675-1679. https://doi.org/10.1038/bjc.2011.138

13. Baselga J, Gelmon KA, Verma S et al (2010) Phase II trial of pertuzumab and trastuzumab in patients with human epidermal growth factor receptor 2-positive metastatic breast cancer that progressed during prior trastuzumab therapy. J Clin Oncol 28:1138-1144. https://doi.org/10.1200/jco.2009.24.2024 
14. Urruticoechea A, Rizwanullah M, Im S-A (2017) et al. Randomized phase iii trial of trastuzumab plus capecitabine with or without pertuzumab in patients with human epidermal growth factor receptor 2positive metastatic breast cancer who experienced disease progression during or after trastuzumabbased therapy. J Clin Oncol 35:3030-3038. https://doi.org/10.1200/jco.2016.70.6267

15. Gianni L, Pienkowski T, Im Y-H et al (2012) Efficacy and safety of neoadjuvant pertuzumab and trastuzumab in women with locally advanced, inflammatory, or early HER2-positive breast cancer (neosphere): a randomised multicentre, open-label, phase 2 trial. Lancet Oncol 13:25-32. https://doi.org/10.1016/s1470-2045(11)70336-9

16. Amiri-Kordestani L, Wedam S, Zhang L et al (2014) First FDA approval of neoadjuvant therapy for breast cancer: pertuzumab for the treatment of patients with HER2-positive breast cancer. Clin Cancer Res 20:5359-5364. https://doi.org/10.1158/1078-0432.ccr-14-1268

17. Modi S, Saura C, Yamashita T et al (2020) Trastuzumab deruxtecan in previously treated HER2positive breast cancer. N Engl J Med 382:610-621. https://doi.org/10.1056/nejmoa1914510

\section{Figures}

\section{Patients, \%}

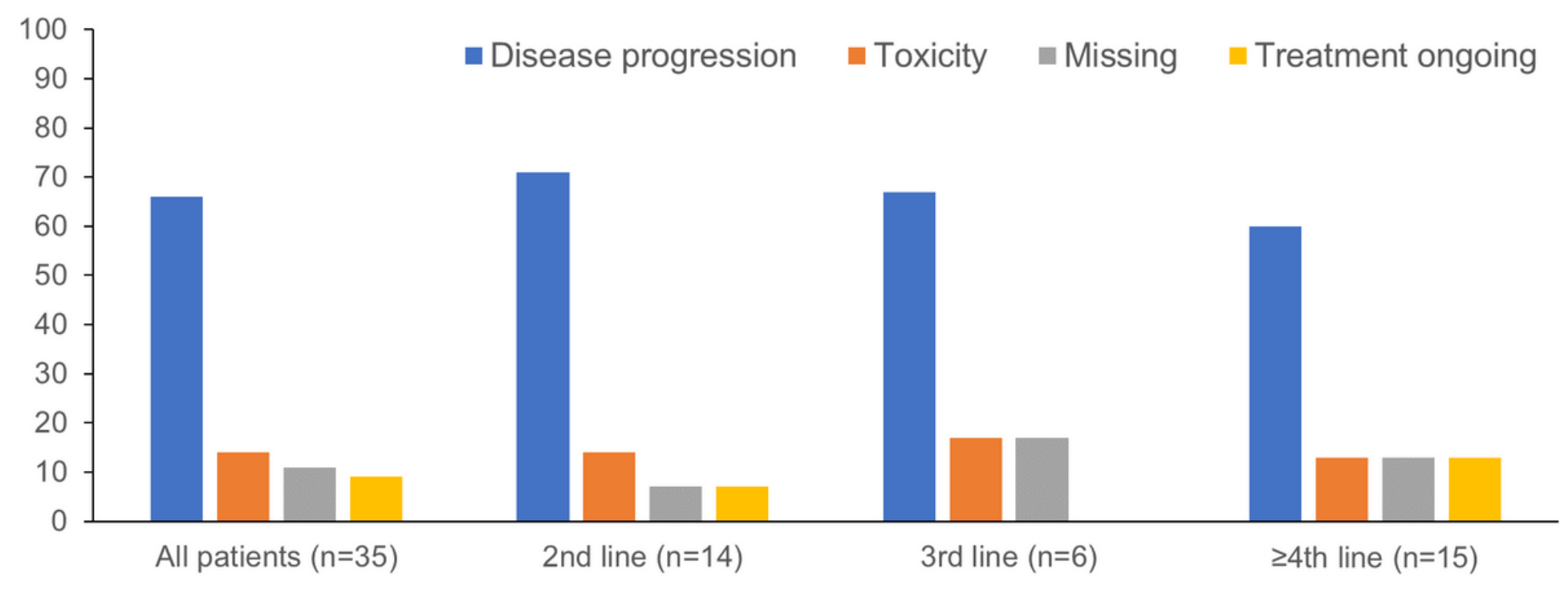

\section{Figure 1}

Treatment discontinuation indications 


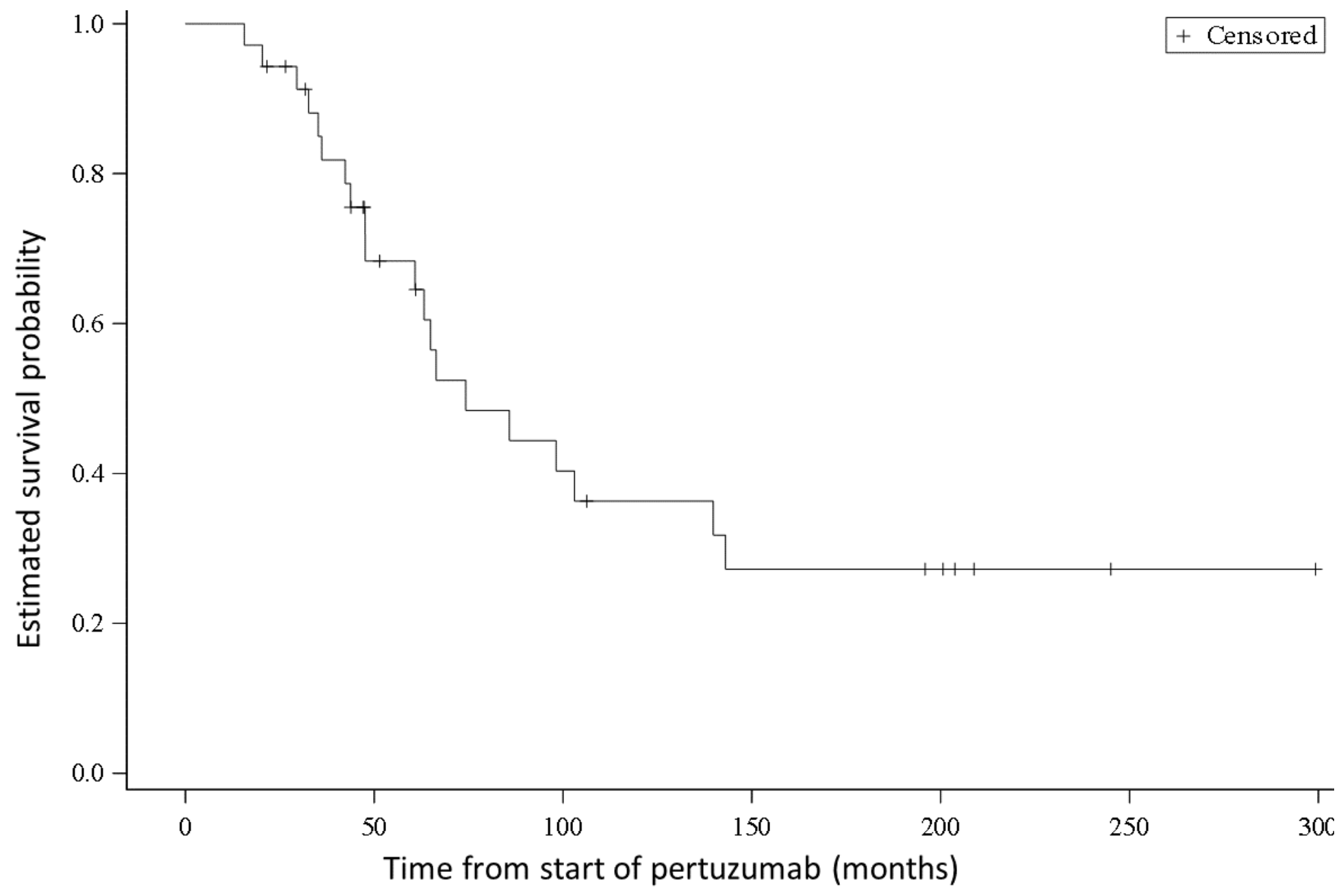

Figure 2

Overall survival 